\title{
Free Energy Correction to Rigid Body Docking : Application to the Colicin E7 and Im7 Complex
}

\author{
Sangwook $\mathrm{Wu}^{1}$, Vasu Chandrasekaran ${ }^{1}$, and Lee G. Pedersen ${ }^{1,2}$ \\ ${ }^{1}$ Department of Chemistry \\ University of North Carolina, \\ Chapel Hill, NC 27599-3290 \\ ${ }^{2}$ Laboratory of Structural Biology, \\ NIEHS, RTP, NC 27709-12233 \\ sangwoow@email.unc.edu, \\ vasu@email.unc.edu, \\ pederse3@niehs.nih.gov
}

\begin{abstract}
We performed a 2-dimensional free energy calculation in the conformational space composed of two structures, best RMSD (Root Mean Square Distance) and the worst RMSD structures using ZDOCK on the Colicin E7 (protein) and Im7 (Inhibitor) complex. The lowest free energy minimum structure is compared to the X-ray crystal structure and the best RMSD docking structure. The free energy correction for the best RMSD structure shows an alternative in the prediction of a flexible loop position, which could not describe rigid body docking.
\end{abstract}

Keywords: Free energy calculation, docking, Colicin E7-Im7 complex.

\section{Introduction}

Docking is a method for estimating the near native structure for a protein-protein complex or a protein-ligand complex through shape or chemical (hydrophobic, hydrophilic) complementarity [12]. For example, the near native structure for a protein-protein complex can be easily found if a complementary shape exists between the interface of two proteins. For an efficient search for the near native docked structure from a set of mostly "incorrectly" docked structures, the docking method performs a rough prediction by treating the two proteins as rigid bodies. Through appropriate rotation and translation of the two rigid bodies using fast Fourier Transforms (FFTs) [3], a score is assigned using a scoring function that depends on how close two proteins fit at the complementary interface. At this stage of rough prediction, the shape information about two entities plays a dominant role. As well as shape information, a docking method may also make use of complementary information provided by electrostatics or hydrophobic interactions at the interface of the protein-protein complex. The information about the chemical complementarity between two protein complexes leads to an energetic correction to the shape complementary based on the FFT technique, 
which is adapted by FTDOCK [4]. For a more elaborate correction of the implicit solvent model, a desolvation energy correction using the ACE (Atomic Contact Energy) [5] is added to the FFT technique and the electrostatic correction in ZDOCK [6]. In addition to electrostatics, the geometry-based hydrophobic complementarity at the interface of protein-protein complex has been incorporated into the FFT-based algorithm in MolFit [7. Recently, more advanced algorithms have been developed to improve the rigid body docking based on FFT through the incorporation of a pairwise structure-based potential in PIPER [8. At the refinement stage of prediction, more elaborate algorithms such as FlexE [9] are adapted into the docking methods to describe flexible side chains or backbone movements through the superimposed structures of the ensemble. Dock 4.0 [10] has been implemented with an incremental construction and random search algorithm. Molecular Dynamics (MD) simulation in an explicit solvent model has been applied to myosin phosphatase targeting subunit (MYPT) and its binding site in protein phosphatase-1 (PP1) 11 with a 2-5 ns simulation and FK506 (ligand)-FKBP (FK binding protein) with a 1 ns simulation [12. However, despite these several algorithms, the incorporation of information about flexible side chain and backbone movements remains one of the main challenges facing the modern docking method. In this study, we propose a free energy correction to the rigid body docking method. The free energy method through effective conformational sampling using the WHAM (Weighted Histogram Analysis Method) procedure 13 14 15 16 17] is able to predict the lowest free energy minimum structure of a protein-protein complex. One of the advantage of the free energy technique in the prediction of near native structure for a protein-protein complex is that it incorporates the dynamics of the protein-protein complex. It can provide us with information of flexible loop movements, depending on the timescale of the MD simulation. In addition, it also provides us with entropy information as well as the energetics of the protein-protein complexes. Such entropy information can prevent the overestimation of the energetics involving the residues at the interface of the protein-protein complex. Entropy is a significant factor which strongly influences the formation of a complex. The general free energy method for predicting protein-protein complex requires a huge amount of sampling in conformational space, especially if starting as a blind trial of the conformational sampling. Such a sampling task becomes aggravated as the protein size increases, and such requires expensive computational expenditure. However, use of rigid body docking, a global search for a "nearly correctly docked structure" from "the set of incorrectly docked structures", could dramatically decrease the burden of conformational sampling for the free energy calculation. In this case, the conformational sampling for the free energy calculation is focused on the region of near native structures for the protein-protein complex identified by rigid body docking. Here, we suggest an efficient method for prediction of protein-protein complexes by combining the free energy method with the docking method. 


\section{Methods}

\subsection{Rigid Body Docking}

The free energy method combined with the docking method is applied to a trial protein-inhibitor system : DNAase domain of Colicin E7 (Protein)-Im7 (Inhibitor) for which we have experimental information. Colicins are protein toxins produced by Escherichia coli [18. The cytotoxic activity is known to be suppressed by binding with its inhibitor [19]. One of the interesting features of colicin and its inhibitors is that the binding affinity is among the strongest known in protein-inhibitor interactions [20]. A test case for the free energy docking technique was selected from the decoy set of the published "Protein-Protein Docking Benchmark" 21]. The benchmark includes individually crystallized receptor and ligand PDBs, along with the co-crystallized complex PDBs for testing protein docking algorithms. The endonuclease domain of Colicin E7 in complex with its inhibitor Im7 protein was used as the test case due to the relatively small size of the protein-protein complex. Two structures were chosen for the references for the free energy calculations based on their RMSDs with respect to the crystallized structure (PDB code : 7CEI). The deviations of $C_{\alpha}$ RMSD values of the docked structures (compared to X-ray crystal structure) generated by ZDOCK is in the range of from $2.12 \AA$ (Best RMSD) to $36.64 \AA$ (Worst RMSD) 1 . The conformations of the two structures, best RMSD and the worst RMSD, is shown in Fig. 1. The other intermediate structures lie between the two extremes that we have chosen.

\subsection{Order Parameter and Free Energy Surface}

We choose the $\mathrm{Q}$ value, the similarity index between two conformations, as an order parameter for the free energy calculation. Q has been widely used in the free energy calculations in protein folding studies [22 23]. It is defined as

$$
Q_{A}=\frac{1}{N} \sum_{i j} \exp \left[-\frac{\left(r_{i j}-r_{i j}^{A}\right)^{2}}{2 \sigma^{2}}\right]
$$

where $r_{i j}$ is the distances between $i$-th and $j$-th atom in conformation of interest, $r_{i j}^{A}$ is the same for the conformation $A$ for which the $Q_{A}$ value is defined, and normalization factor $N$ is equal to number of pairs of atoms whose positions define the conformation. The similarity index $Q_{A}$ changes from 1 (for the conformation $A$ ) to 0 (for a conformation with no resemblance to $A$ ). Normally, only $C_{\alpha}$ carbons are chosen in the calculation of the $Q$ value. To track the flexible protein movements, however, we extend the range of atoms to include $C_{\beta}, C_{\gamma}$, $C_{\delta}, C_{\varepsilon}$ and $C_{Z}$ atoms [24. In Eq. 1, $\sigma$ controls the resolution of the $Q$ value. Considering that the resolution of the X-ray crystal of 7CEI is $2.3 \AA[19, \sigma$ is set to $2 \AA$ in our study. A total of 878 atoms are considered in the calculation

\footnotetext{
${ }^{1}$ The RMSD values were calculated after the alignment of the $\operatorname{Im} 7$ (Residue 1 to 87 )
} using VMD. 


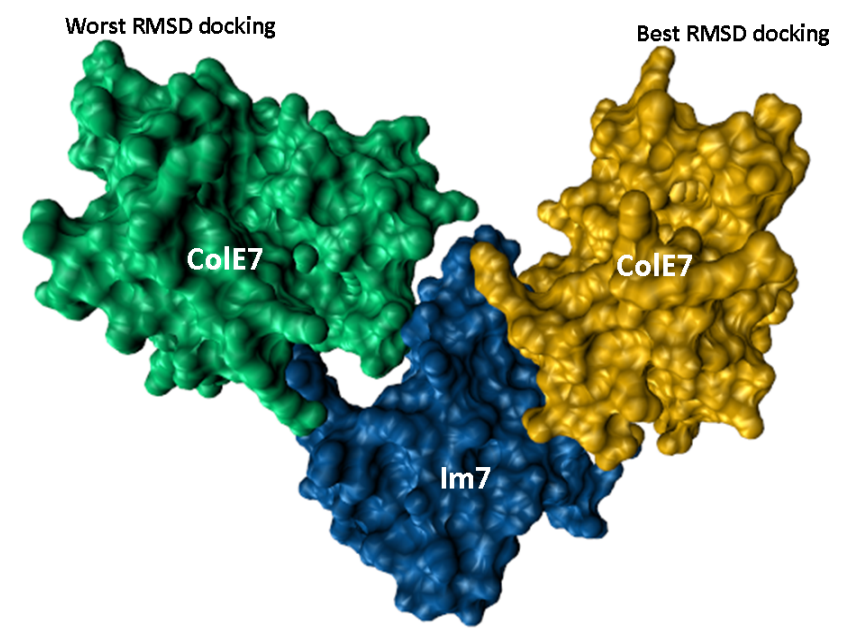

Fig. 1. The best RMSD (Yellow) and worst RMSD (Green) docking structures. The cognate inhibitor, $\operatorname{Im} 7$, is in common and shown as a blue color. Yellow represents the DNAase domain of Colicin E7 in the best RMSD structure and green represents the DNAase domain of Colicin E7 in the worst RMSD structure.

of $Q_{A}$ value; this definition is essential in tracking the conformational change which involve small movements or rotations of flexible side chains. We choose two references, the best docked structure and the worst docked structure as shown in Fig. 1. The basic idea is to perform all the conformational sampling using WHAM between these two extreme references.

\subsection{Simulation}

We performed free energy calculations in the 2-dimensional conformation space composed of ( $\left.Q_{\text {bestRMSD }}, Q_{\text {worstRMSD }}\right)$. The conformational sampling is guided by a biasing potential,

$$
V\left(Q_{\text {best }}, Q_{\text {worst }}\right)=\frac{1}{2} k_{\text {best }}\left(Q_{\text {best }}-Q_{\text {best }}^{\min }\right)^{2}+\frac{1}{2} k_{\text {worst }}\left(Q_{\text {worst }}-Q_{\text {worst }}^{\min }\right)^{2}
$$

where $k_{\text {best }}$ and $k_{w o r s t}$ are spring constants and $Q_{b e s t}^{\min }$ and $Q_{w o r s t}^{\min }$ are the locations at which the biasing potentials are applied. The spring constants $k_{\text {best }}$ and $k_{w o r s t}$ are in the range of from $11.5 \mathrm{kcal} / \mathrm{mol} / \AA^{2}$ to $82.5 \mathrm{kcal} / \mathrm{mol} / \AA^{2}$. These spring constants are determined so as to obtain the best overlap between trajectories for good sampling. For dielectric constant $\epsilon=80.0$, a total of 367 windows are used for each different $Q_{\text {worst }}^{\min }$ and $Q_{\text {best }}^{\min }$ ranging from 0.5 to 1 . Each window was run for $50 \mathrm{ps}$. For productive data, the first $10 \mathrm{ps}$ simulation is removed. Thus, the total sampling corresponds to $14.7 \mathrm{~ns}$ (40 ps $\times 367$ windows). On the other hand, for dielectric constant $\epsilon=4.0$, a total of 388 windows are used for each different $Q_{\text {worst }}^{\min }$ and $Q_{b e s t}^{\min }$ ranging from 0.5 to 1 . Each window was run for 100 ps. For productive data, the first 10 ps simulation is removed. Thus, the 
total sampling corresponds to 32.8 ns (90 ps $\times 364$ windows). All of the MD simulations were performed using LAMMPS (Large-scale Atomic and Molecular Massively Parallel Simulator) at the atomistic level with the CHARMM27 protein-lipid force field [25]. The best RMSD structure and the worst RMSD structures were treated with a dielectric constant of 80.0 and 4.0 using the distance dependent dielectric solvent model, $\epsilon(r)=\epsilon r$. The two references, the best RMSD and the worst RMSD structures, were minimized using the steepest descent gradient method in NAMD employing the CHARMM force field. The charm2lammps perl script 25] converted each minimized structures into the initial structures for LAMMPS. These were equilibrated for 1 ns at $293 \mathrm{~K}$. In the process of equilibration at $293 \mathrm{~K}$, target Molecular Dynamics was performed for the two structures to keep the two structures less than $0.1 \AA$ of the backbone RMSD with respect to the two references. The Coulombic and Lennard-Jones interactions were calculated with a 10.0/12.0 $\AA$ twin-range cutoff. This is a feasibility study; the validity of the method is to be established (below). The various computational compromises can be removed in future work.

\section{Result}

Figure 2 shows that the 2 -dimensional free energy surface (FES). ( $Q_{\text {best }}, Q_{\text {worst }}: 1.0$, $0.59)$ and $\left(Q_{\text {best }}, Q_{\text {worst }}: 0.59,1.0\right)$ corresponds to the best RMSD and the worst RMSD structures respectively. It is gratifying that the lowest free energy structure at $\epsilon=4.0$ is quite similar to the X-ray crystal structure with $C_{\alpha}$ RMSD of $2.05 \AA$. Figure 3 . a shows the superimposed images of best RMSD (Cyan) and the lowest free energy minimum structure (Orange). Figure 3.b shows the superimposed images of the the lowest free energy minimum structure (Orange) and the X-ray crystal (Green). The main difference at the backbone level between the lowest free energy structure and X-ray structure lies in loop configurations from

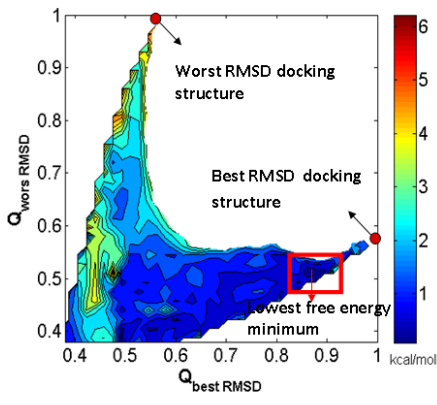

Dielectric constant : $\mathbf{8 0 . 0}$

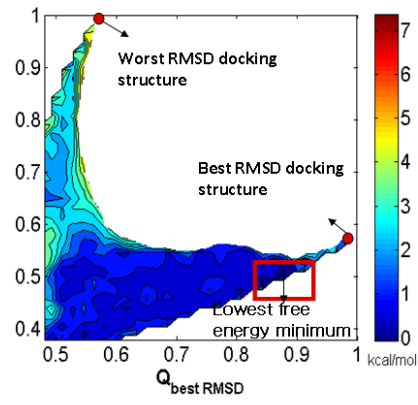

Dielectric constant : 4.0

Fig. 2. 2-dimensional free energy surface of ColE7-Im7 complex for dielectric constant $\epsilon=80.0$ and $\epsilon=4.0$. The two red circles are best RMSD docking structure and worst docking RMSD structure. The red box corresponds to the lowest free energy minimum $\left(Q_{\text {best }}, Q_{\text {worst }}: 0.878,0.504\right)$ at $\epsilon=80.0$ and $\left(Q_{\text {best }}, Q_{\text {worst }}: 0.894,0.520\right)$ at $\epsilon=4.0$. 


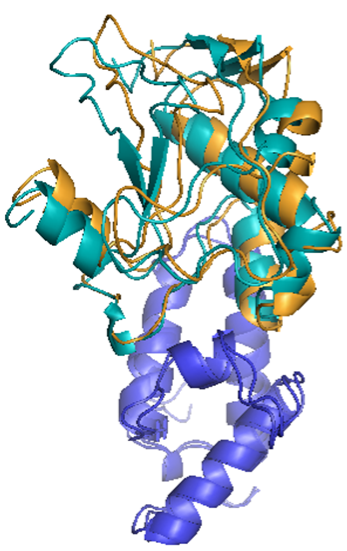

a

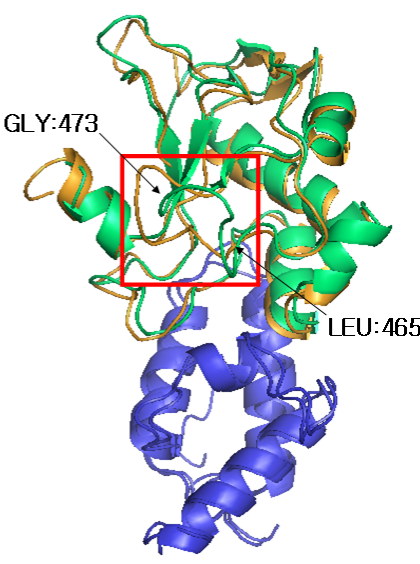

b

Fig. 3. Figure 3.a shows the superimposed images of best RMSD (Cyan) and the lowest free energy minimum structure (Orange) at dielectric constant $\epsilon=4$.0. Figure 3.b shows the superimposed images of the the lowest free energy minimum structure (Orange) and the X-ray crystal (Green) dielectric constant $\epsilon=4.0$. The $\operatorname{Im} 7$ (Inhibitor) is shown as blue color.

Leu465 to Gly473 in red box in Fig. 3b. The buried solvent accessible surface areas (SASA) were calculated for the X-ray crystal structure $\left(1381 \AA^{2}\right)$, the best RMSD docking structure $\left(1434 \AA^{2}\right)$, and the lowest free energy minimum structure at $\epsilon=4.0\left(1787 \AA^{2}\right) \sqrt{2}$. It indicates the lowest free energy minimum structure is more tightly packed at the interface of the ColE7-Im7 complex than the X-ray crystal structure.

\section{Discussion}

We performed free energy calculation in the 2-dimensional conformation space composed of the two references, best and the worst RMSD structures. The docking method and the free energy sampling method are quite complementary to each other. The docking method (based on the rigid body docking) dramatically narrows the sampling space. Otherwise, the blind test would require exhaustive computational performance. If the X-ray crystal structure of the protein-protein complexes is identified (7CEI in our study), we can test the validity of the docking method. By calculating the RMSD value with respect to the X-ray crystal structure, we can choose two references of the best and worst RMSD docking structures. Also, if we also have biological information (such as catalytic site or active site), physical (main groove or minor grove) and chemical information (hydrophobic, hydrophilic, H-bonds) about the protein-protein complex, the docking method remains an essential tool to predict the "nearly correct" configuration of

${ }^{2} 1.4 \AA$ was used as the probe radius for the calculation of SASA. 
the protein-protein complexes within the rigid body approximation. However, if the X-ray crystal form of the protein-protein complex is not available, or when the sufficient information about the chemical and physical composition of each entity in protein-protein complexes is missing, the rigid docking method would be less reliable. Furthermore, when flexible loops play critical roles in forming protein-protein complexes, the error in the rigid body prediction will increase. In this case, however, the docking method provide several "plausible" candidates for the protein-protein complex at the rough prediction level. The free energy method then performs the key mission of finding the "nearly correct structure" from the "plausible" structures, including information of flexible loops. As a future study, we will apply our method with explicit water to the present case and finally to the case for which an X-ray crystal of the complex has not been solved.

\section{Acknowledgements}

LGP acknowledges support from NIH-06350, NSF FRG DMR 0804549, the intramural research program of the NIH and National Institute of Environmental Health Sciences.

\section{References}

1. Smith, G.R., Stenberg, M.J.E.: Prediction of Protein-Protein Intreactions by Docking Methods. Curr. Opin. Struct. Biol. 12, 28-35 (2002)

2. Ritchie, D.W.: Recent Progress and Future Directions in Protein-Protein Docking. Curr. Prot. Pepti. Sci. 9, 1-15 (2008)

3. Katchalski-Katzir, E., Sharive, I., Eisenstein, M., Friesem, A.A., Aflalo, C., Vakser, I.A.: Molecular Surface Recognition: Determination of Geometric Fit between Proteins and Their Ligands by Correlation Technique. Proc. Natl. Acad. Sci. USA. 89, 2195-2199 (1992)

4. Gabb, H.A., Jackson, R.M., Sternberg, M.J.E.: Modelling Protein Docking Using Shape Complementarity, Electrostatics and Biochemical Information. J. Mol. Biol. 272, 106-120 (1997)

5. Zhang, C., Vasmatzis, G., Cornette, J.L., DeLisi, C.: Determination of Atomic Desolvation Energies from the Structures of Crystallized Proteins. J. Mol. Biol. 267, 707-726 (1997)

6. Chen, R., Weng, Z.: Docking Unbound Proteins Using Shape Complementarity, Desolvation, and Electrostatics. Proteins 47, 281-294 (2002)

7. Berchanski, A., Shapira, B., Eisenstein, M.: Hydrophobic Complementarity in Protein-Protein Docking. Proteins 56, 130-142 (2004)

8. Kozakov, D., Brenke, R., Comeau, S.R., Vajda, S.: PIPER: an FFT-Based Protein Docking Program with Pairwise Potentials. Proteins 65, 392-406 (2006)

9. Claußen, H., Buning, C., Rarey, M., Lengaue, T.: FlexE:Efficeient Molecular Docking Considering Protein Structure Variations. J. Mol. Biol. 308, 377-395 (2001)

10. Ewing, T.J.A., Makino, S., Skillman, A.G., Kuntz, I.D.: DOCK 4.0:Search Strategies for Automated Molecular Docking of Flexible Molecular Databases. J. Comput. Aided. Mol. Des. 15, 411-428 (2001) 
11. Smith, G.R., Fitzjohn, P.W., Page, C.S., Bates, P.A.: Incorporation of Flexibility into Rigid-Body Docking: Applications in Rounds 3-5 of CAPRI. Proteins 60, 263 268 (2005)

12. Zacharias, M.: Rapid Protein-Ligand Docking Using Soft Modes from Molecular Dynamics Simulations to Account for Protein Deformability:Binding of FK506 to FKBP. Proteins 54, 759-767 (2004)

13. Roux, B.: The Calculation of the Potential of Mean Force Using Computer Simulation. Comput. Phys. Comm. 91, 275-282 (1994)

14. Kumar, S., Rosenberg, J.M., Bouzida, D., Swendsen, R.H., Kollman, P.A.: The Weighted Histogram Analysis Method for Free-Energy Calculation on Biomolecules. I. The Method. J. Comput. Chem. 13, 1011-1021 (1992)

15. Ferrenberg, A.M., Swendsen, R.H.: Optimized Monte Carlo Data Analysis. Phys. Rev. Lett. 63, 1195-1198 (1989)

16. Banavali, N.K., Roux, B.: Free Energy Landscape of A-DNA to B-DNA Conversion in Aqueous Solution. J. Am. Chem. Soc. 127, 6866-6876 (2005)

17. Arora, K., Brooks III, C.L.: Large-Scale Conformational Transitions of Adenylate Kinase Appear to Involve a Population-Shift Mechanism. Proc. Natl. Acad. Sci. USA 104, 18496-18501 (2007)

18. Pugsley, A.P., Oudega, B.: Methods for Studing Colicins and Their Plasmids. In: Hardy, K.G. (ed.) Plasmids: A Practical Approach, pp. 105-161. IRL Press, Oxford $(1987)$

19. Ko, T.P., Liao, C.C., Ku, W.Y., Chak, K.F., Yuan, H.S.: The Crystal Structure of the DNAase Domain of Colicin E7 in Complex with its Inhibitor Im7 Protein. Struct. 7, 91-102 (1999)

20. Wallis, R., Leung, K.Y., Pommer, A.J., Videler, H., Moor, G.R., James, R., Kleanthous, C.: Protein-Protein Interactions in Colicin E9 DNAase-Immunity Protein Complexes.2. Cognate and Noncognate Interactions That Span the Millimolar to Femtomolar Affinity Range. Biochem. 34, 13751-13759 (1995)

21. Mintseris, J., Wiehe, K., Pierce, B., Anderson, R., Chen, R., Janin, J., Weng, Z.: Protein-Protein Docking Benchmark 2.0: An Update. Proteins 60, 214-216 (2005)

22. Wolynes, P.G.: Landscapes, Funnesls, Glasses, and Folding: from Metaphors to Software. Proc. Am. Phil. Soc. 145, 555-563 (2001)

23. Takagi, F., Koga, N., Takada, S.: How Protein Thermodynamics and Folding Mechanisms are Altered by the Chaperonin Cage:Molecular Simulations. Proc. Natl. Acad. Sci. USA 100, 11367-11372 (2003)

24. Wu, S., Zhuravlev, P.I., Papoian, G.A.: High Resolution Approach to the Native State Enesemble Kinetics and Thermodynamics. Biophys. J. 95, 5524-5532 (2008)

25. Plimton, S.J.: Fast Parallel Algorithms for Short-Range Molecular Dynamics. J. Comput. Phys. 117, 1-19 (1995) 